\title{
Neutron monitor yield function at several altitudes above sea level: new improved computation
}

\author{
Alexander Mishev* \\ Space Climate Research Unit, University of Oulu, Finland; Sodankylä Geophysical Observatory, \\ University of Oulu, Finland. \\ E-mail: alexander.misheveoulu.fi
}

Gennady Kovaltsov

Ioffe Physical-Technical Institute of Russian Academy of Sciences, St. Petersburg, Russia.

Ilya Usoskin

Space Climate Research Unit, University of Oulu, Finland; Sodankylä Geophysical Observatory, University of Oulu, Finland.

E-mail: ilya.usoskin@oulu.fi

For an analysis of solar particle events using neutron monitor data it is necessary to model the global neutron monitor network response. This is possible using the corresponding yield function(s). We present new improved computations of standard 6NM64 yield functions for primary protons and alpha particles. The yield functions were computed at several depths, encompassing all the historical and existing neutron monitors. The computations were carried out with the Planetocosmics Monte-Carlo tool for extensive air shower simulations. All the secondary particles, which contribute to the count rate of a NM were considered. An effect of the geometrical correction of the NM effective area was also considered above 5-10 GeV/nucleon. The new NM yield function is compared with previous estimates and with experimental altitude and latitude surveys. The new NM yield function was applied to an analysis of ground level enhancement on the basis of global NM network data. The application of previously used double attenuation length method and the new yield function for ground level enhancement analysis are compared.

36th International Cosmic Ray Conference - ICRC 2019-

24 July-1 August, 2019

Madison, United States

\footnotetext{
${ }^{*}$ Speaker.
} 


\section{Introduction}

Systematic study of solar energetic particles (SEPs) provides a reliable basis to understand their acceleration mechanism, propagation in the interplanetary space and quantification $[1,2,3$, $4,5]$. As a result of solar eruption(s) e.g. solar flare(s) and/or coronal mass ejection (CME), SEPs can be accelerated to several tens of $\mathrm{MeV} /$ nucleon $[6,7]$. In some cases, SEPs are accelerated to energy exceeding $100 \mathrm{MeV} /$ nucleon or even to a $\mathrm{GeV}$ range. In such cases the SEP energy is high enough to generate a cascade process in the Earth's atmosphere. Secondary particles of the cascade reach the ground and can be registered by ground based detectors e.g. neutron monitors (NMs). This class of events is called ground-level enhancements (GLEs) [8, 9]. Over the years GLEs have been routinely studied using NM records. NMs data analysis is usually used to derive spectral and angular characteristics of SEPs in the vicinity of Earth by modelling the global NM network response $[10,11]$. For this purpose it is necessary to possess precise information of SEP propagation in the Earth's atmosphere and NM efficiency for registration of given secondary particles. The NM specific yield function incorporates the full complexity of the atmospheric cascade development, the secondary particle propagation in the atmosphere and the registration efficiency of the detector itself [12]. At recent, application of Monte Carlo methods allowed one to compute realistically the specific NM yield function $[12,13,14,15,16]$. Newly computed by us NM yield function, which considers explicitly a geometrical correction related to the finite lateral extend of the secondary particles, was shown to be consistent with latitude surveys and was recently validated $[17,18,19]$.

Nowadays, the global worldwide NM network consists of about 50 stations. An essential part of those NMs is located at moderate and high level altitude (e.g. $>500 \mathrm{~m}$ above sea level), therefore they are more sensitive, specifically to SEPs, because the reduced atmospheric particle attenuation compared to sea level ones (Fig.1). Moreover, a large number of high-altitude NMs have been used for continuous recordings of cosmic ray (CR) intensity, whose data about GLEs are accordingly stored in the International GLE database https: / / gle.oulu.fi [20].

During the analysis of GLEs, usually the NM count rate increase are normalized to sea level by employing the two attenuation lengths method [21]. This would include some uncertainty, mostly due to the assumption of SEP spectrum slope and is not suitable for operational space weather purposes [22]. Therefore, computation of NM yield function at several altitudes, which encompass all historical and in operation NMs is rather important. Here, we computed NM yield function for a standard 6NM64 at various altitudes similarly to [15].

\section{Neutron monitor yield function at different altitudes}

The response of a NM to cosmic rays (CRs) is modelled using the expression:

$$
N\left(P_{c}, h, t\right)=\sum_{i} \int_{P_{c}}^{\infty} S_{i}(P, h) J_{i}(P, t) d P
$$

where $P_{c}$ is the local geomagnetic cut-off rigidity [23], $h$ is the atmospheric depth, $S_{i}(P, h)\left[\mathrm{m}^{2} \mathrm{sr}\right]$ is the specific NM yield function for primaries of particle type $i$ (protons, $\alpha$-particles, heavy nuclei), $J_{i}(P, t)\left[\mathrm{GV} \mathrm{m}^{2} \mathrm{sr} \mathrm{sec}^{-1}\right.$ is the rigidity spectrum of primary particle of type $i$ at time $t$. Accordingly 


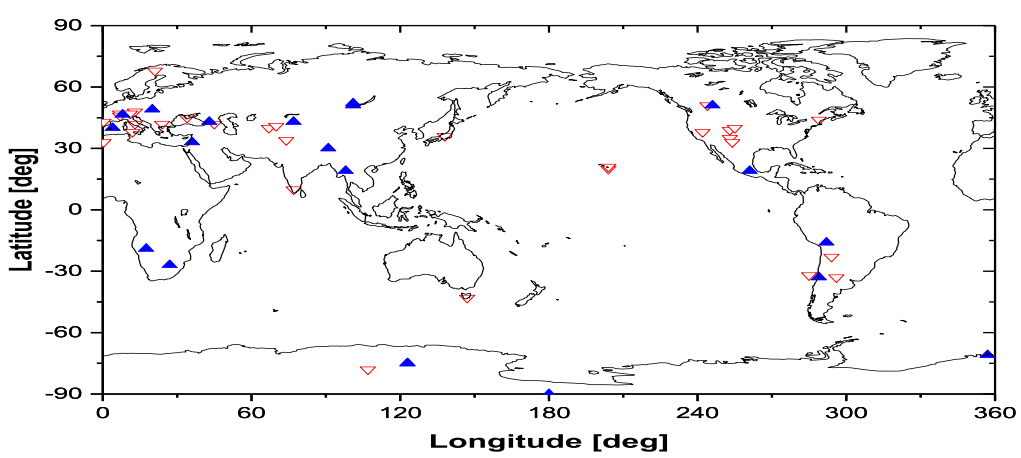

Figure 1: Location map of the non sea level NMs. Up blue triangles correspond to stations in use, while down red triangles to closed stations.

the NM yield function is defined as:

$$
S_{i}(P(E), h)=\sum_{j} \iint A_{i}(E, \theta, C(E)) \cdot F_{i, j}(P, h, E, \theta) d E d \Omega
$$

where $C(E)$ is a geometrical correction factor, which considers the finite lateral expansion of the secondary particles in the cascade, defined according to [15] in the $A_{i}(E, \theta)$, which is the detector effective area and includes the registration efficiency, $F_{i, j}$ is the flux of secondary particles with energy $E$ and angle of incidence $\theta$. The relative count rate increase of a given NM during GLE is given as:

$$
\frac{\Delta N(P)}{N}=\frac{\frac{1}{13} \sum_{k} \int_{P_{\text {cut }}}^{P_{\max }} J_{\text {sep }}(P, t) S_{k}(P) G(\alpha(P, t)) d P}{\int_{P_{\text {cut }}}^{\infty} J_{G C R}(P, t) Y(P) d P}
$$

where $J_{\text {sep }}$ is the rigidity spectrum of SEPs, $J_{G C R}(P, t)$ is the rigidity spectrum of GCR at given time $t, G(\alpha(P, t))$ is the pitch angle distribution of SEPs, $N$ is the count rate due to GCR, $\Delta N\left(P_{\text {cut }}\right)$ is the count rate increase due to solar particles, $P_{c u t}$ is the minimum rigidity cut-off of the station, accordingly $P_{\max }=20 \mathrm{GV}$ is the maximum rigidity of SEPs considered in the model, $S_{k}$ is the specific NM yield function for $\mathrm{k}=0^{\circ}, 15^{\circ}, 30^{\circ}$ and $45^{\circ}$, which accounts the contribution of oblique events [24] from 13 weighted by solid angle different segments (Fig. 2), which is particularly important for modelling strong and/or very anisotropic events. Expression (2.3) allows one to model the global NM network response and to derive the spectral and angular characteristics of SEPs using a convenient optimization [25, 26, 27], explicitly considering obliqueness of the events and NM responses at different altitudes $[28,29,30]$. Note, that in case of weak events and/or during isotropic phase of an event, the $S_{k}$ can be replaced in (2.3) with isotropic NM yield function, which considerably simplifies the computations, but lead to comparable results [28].

For computations of the specific NM yield function we performed Monte Carlo simulations of $\mathrm{CR}$ induced atmospheric cascades due to primary protons and $\alpha$-particles with energy in a wide range. Propagation and interaction of primaries in the Earth's atmosphere was simulated with PLANETOCOSMICS [31] code, employing NRLMSISE 00 atmospheric model [32]. Correction similar to [15] was also considered, which slightly varied as a function of the altitude above sea 


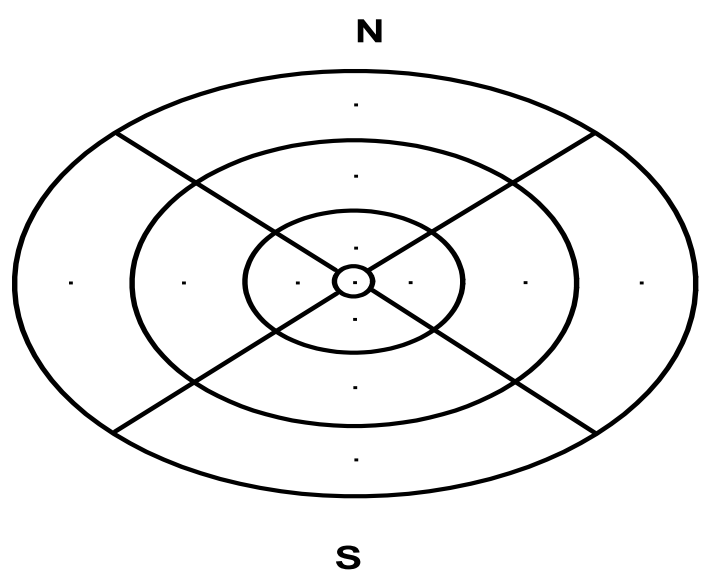

Figure 2: Thirteen segments above a CRs contribute to NM responses. Circles represent zenith angles of $0^{\circ}, 15^{\circ}, 30^{\circ}$ and $45^{\circ} . S$, accordingly $\mathrm{NM}$ viewing cones are computed for each direction market with dots (zenith angles $0^{\circ}, 15^{\circ}, 30^{\circ}$ and $45^{\circ}$ and azimuths $0^{\circ}, 90^{\circ}, 180^{\circ}$ and $270^{\circ}$ ).

level. Thus, we computed the specific NM yield function at several altitudes and for different angles of incidence, namely for isotropic, vertical, $15^{\circ}, 30^{\circ}$ and $45^{\circ}$.

An example of the computation is given in Fig,3, where isotropic $S$ are presented separately for protons and $\alpha$-particles (panel a) as well as a comparison with other computations (panel b) [14].
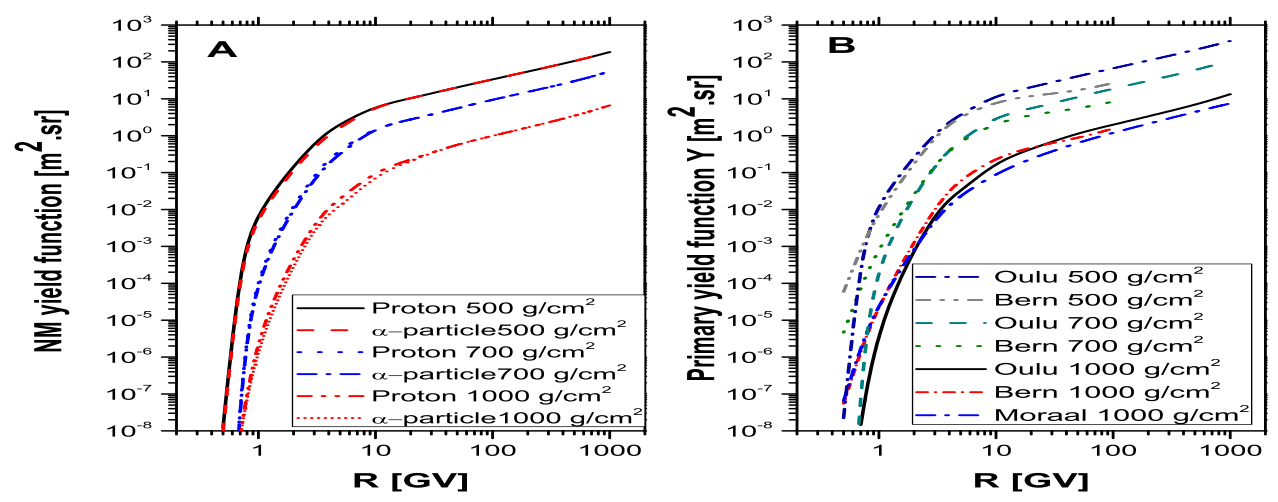

Figure 3: NM yield function for particles with isotropic incidence at different atmospheric depths. Panel a represents NM yield function $S$ for protons and $\alpha$-particles; panel b represents a comparison of $S$ for primary protons with other computations [14].

One can see the relatively good agreement of this work specifically with Bern model [14], particularly in the range of maximal NM response (Fig. 4). The computation corresponding to 


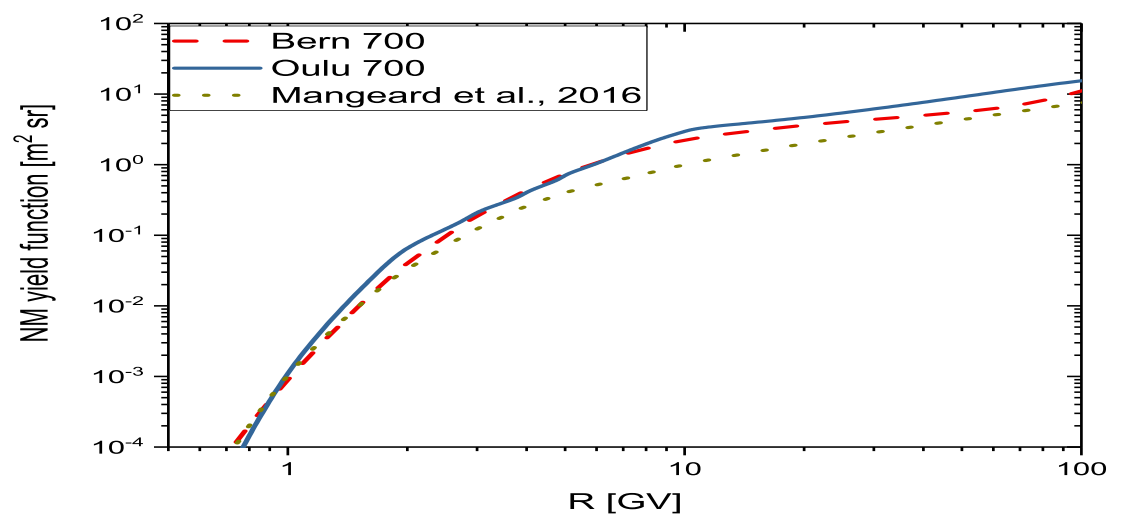

Figure 4: Comparison of several computation of NM yield function $S$ at depth of $700 \mathrm{~g} \cdot \mathrm{m}^{-2}$. Oulu 700 corresponds to this work, Bern 700 to [14], Mangeard 2016 to [16].

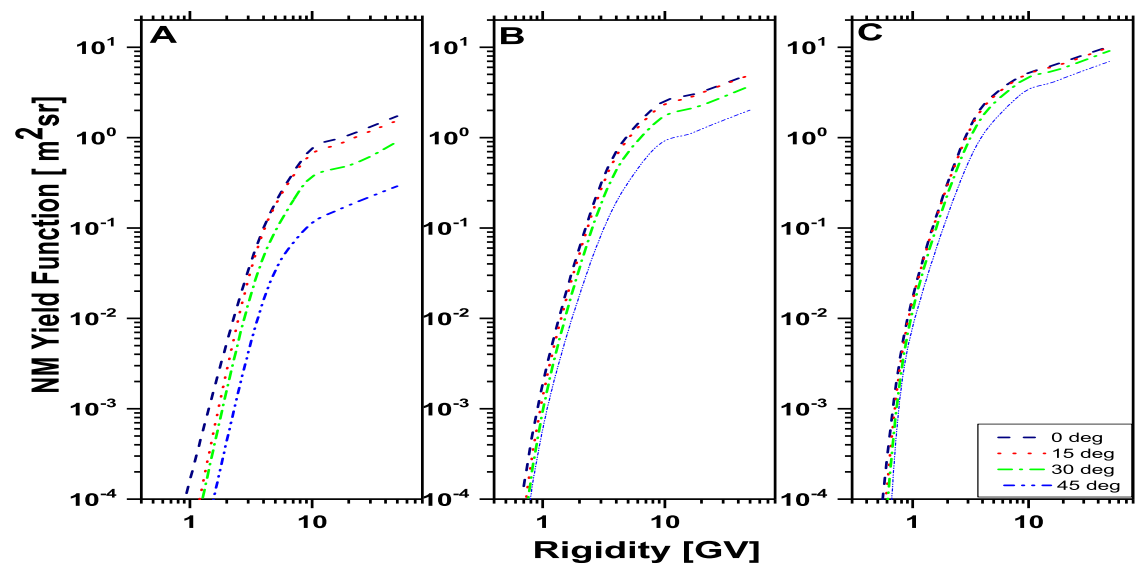

Figure 5: NM yield function $S$ for protons with various incidence. Panel a corresponds to sea level, panel b to $700 \mathrm{~g} \cdot \mathrm{m}^{-2}$ and panel c to $500 \mathrm{~g} \cdot \mathrm{m}^{-2}$, respectively.

oblique events are shown in Fig. 5. Note, that those computations were carried out up to $20 \mathrm{GeV} / \mathrm{n}$, because they aimed particularly SEPs.

\section{Applications}

Reliable analysis of several GLE have been performed using the newly computed specific NM yield functions, explicitly considering the altitude above sea level of the station, i.e., the response of each NM is modelled with a yield function corresponding to it's exact altitude [28, 30]. Moreover, due to the reduced uncertainties and robust procedure, the application of specific high-altitude NM yield function(s) for GLE analysis, allowed us to derive spectra and angular distribution of new sub-class SEPs events, namely sub-GLEs [9], the details are given elsewhere [29] and to derive more precise GLE spectra. 
In addition, we compared the derived SEP spectral and angular characteristics of GLE 71 using two attenuation lengths method [27] and employing NM yield function at several altitudes. During the new analysis the responses of all high-mountain NMs, namely South Pole, which recorded a notable NM count rate increase, accordingly Alma Ata, Baksan, Jungfrau Joch with marginal or null NM count rate increases, were modelled using yield functions corresponding to their exact altitude above sea level. The derived on the basis of the new analysis SEP spectra are with reduced uncertainties compared to rescaling method, the details are given elsewhere. Moreover, we expanded considerably the time span of derived SEP spectra, specifically in the late phase of the event, where an isotropisation of SEPs was observed.

\section{Conclusion}

Here, we presented new improved computations of standard NM 64 yield function at several altitudes. The newly yield function was computed for primary protons and $\alpha$-particles. Note, that $\alpha$-particles effectively consider all heavy species of primary CRs [33, 34]. The computations were performed using a realistic atmospheric model employing Monte Carlo simulations. Similarly to sea level NM yield function, a geometrical correction of the detector effective area was explicitly considered, which was found slightly to vary as a function of altitude above sea level. This correction explicitly takes into account the finite lateral extend of the CR-induced atmospheric cascade. It becomes important at energies greater of about 5-10 GeV/nucleon. Hence, we computed NM yield functions at various altitudes, which appeared consistent with the experimental NM count rates for several NM stations. In addition, we computed NM yield function for events with different incidence, namely $0^{\circ}, 15^{\circ}, 30^{\circ}$ and $45^{\circ}$, which allowed us to consider several important effects and to model more precisely, specifically very strong and highly isotropic, events. The newly improved computation of the NM yield function at several altitudes, improved the developed by us procedure for GLE analysis using NM data, leading to a faster convergence of the optimization and more robust results in contrast to procedure based on rescaling NM responses to sea level.

\section{Acknowledgements}

This work was supported by the Academy of Finland (project 307411, Center of Excellence ReSoLVE, HEAIM project 314982 and 316223) and by the International Space Science Institute support to International Team 441: High EneRgy sOlar partICle Events Analysis (HEROIC).

\section{References}

[1] M. Aschwanden, GeV particle acceleration in solar flares and ground level enhancement (GLE) events, Space Science Reviews 171 (2012), no. 1-4 3-21.

[2] N. Gopalswamy, S. Yashiro, N. Thakur, P. Mäkelä, H. Xie, and S. Akiyama, The 2012 July 23 backside eruption: An extreme energetic particle event?, Astrophysical Journal 833 (2016), no. 2216.

[3] K.-L. Klein and S. Dalla, Acceleration and propagation of solar energetic particles, Space Science Reviews 212 (2017), no. 3-4 1107-1136. 
[4] L. Kocharov, S. Pohjolainen, A. Mishev, M. Reiner, J. Lee, T. Laitinen, L. Didkovsky, V. Pizzo, R. Kim, A. Klassen, M. Karlicky, K.-S. Cho, D. Gary, I. Usoskin, E. Valtonen and R. Vainio, Investigating the origins of two extreme solar particle events: Proton source profile and associated electromagnetic emissions, Astrophysical Journal 839 (2017), no. 279.

[5] L. Kocharov, S. Pohjolainen, M. Reiner, A. Mishev, H. Wang, I. Usoskin and R. Vainio, Spatial organization of seven extreme solar energetic particle events, Astrophysical Journal Letters $\mathbf{8 6 2}$ (2018), no. 2 L20.

[6] R. Vainio, L. Desorgher, D. Heynderickx, M. Storini, E. Flückiger, R. Horne, G. Kovaltsov, K. Kudela, M. Laurenza, S. McKenna-Lawlor, H. Rothkaehl and I. Usoskin, Dynamics of the Earth's particle radiation environment, Space Science Reviews 147 (2009), no. 3-4 187-231.

[7] M. Desai and J. Giacalone, Large gradual solar energetic particle events, Living Reviews in Solar Physics 13 (2016), no. 13.

[8] M. A. Shea, I. Zaljubovsky, M. Wada and A. Inoue, A suggested standardized format for cosmic ray ground-level event data, in Proc. of 19th ICRC La Jolla, USA, 11 - 23 August 1985, vol. 5, pp. 510-513, 1985.

[9] S. Poluianov, I. Usoskin, A. Mishev, A. Shea and D. Smart, GLE and sub-GLE redefinition in the light of high-altitude polar neutron monitors, Solar Physics 292 (2017), no. 11176.

[10] M. Shea and D. Smart, Possible evidence for a rigidity-dependent release of relativistic protons from the solar corona, Space Science Reviews 32 (1982) 251-271.

[11] A. Mishev and I. Usoskin, Computations of cosmic ray propagation in the Earth's atmosphere, towards a GLE analysis, Journal of Physics: Conference Series 409 (2013) 012152.

[12] J. Clem and L. Dorman, Neutron monitor response functions, Space Science Reviews 93 (2000) 335-359.

[13] E. Vashenyuk, Y. Balabin and P. Stoker, Responses to solar cosmic rays of neutron monitors of a various design, Advances Space Research 40 (2007), no. 3 331-337.

[14] E. Flükiger, E. Moser, E. Pirard, R. Butikofer and L. Desorgher, A parameterized neutron monitor yield function for space weather applications, in Proc. of 30th ICRC Merida, Yacatan, Mexico, 3 -11 July 2007, vol. 1, pp. 289-292, 2008.

[15] A. Mishev, I. Usoskin and G. Kovaltsov, Neutron monitor yield function: New improved computations, Journal of Geophysical Research 118 (2013) 2783-2788.

[16] P.-S. Mangeard, D. Ruffolo, A. Sáiz, W. Nuntiyakul, J. Bieber, J. Clem, P. Evenson, R. Pyle, M. Duldig and J. Humble, Dependence of the neutron monitor count rate and time delay distribution on the rigidity spectrum of primary cosmic rays, Journal of Geophysical Research: Space Physics 121 (2016), no. 12 11,620-11,636.

[17] A. Gil, I. Usoskin, G. Kovaltsov, A. Mishev, C. Corti and V. Bindi, Can we properly model the neutron monitor count rate?, J. Geophys. Res. 120 (2015) 7172-7178.

[18] W. Nuntiyakul, A. Sáiz, D. Ruffolo, P.-S. Mangeard, P. Evenson, J. Bieber, J. Clem, R. Pyle, M. Duldig and J. Humble, Bare neutron counter and neutron monitor response to cosmic rays during a 1995 latitude survey, Journal of Geophysical Research: Space Physics 123 (2018), no. 9 $7181-7195$. 
[19] S. A. Koldobskiy, V. Bindi, C. Corti, G. A. Kovaltsov and I. G. Usoskin, Validation of the Neutron Monitor Yield Function Using Data from AMS-02 Experiment 2011-2017, J. Geophys. Res. (Space Phys.) 124 (2019).

[20] I. Usoskin, A. Ibragimov, M. Shea and D. Smart, Database of ground level enhancements (GLE) of high energy solar proton events, Proceedings of Science, Proc. of 34th ICRC Hague, Netherlands, 30 July - 6 August 2015 (2015) 054.

[21] K. McCracken, The cosmic ray flare effect, 1, some new methods for analysis, Journal of Geophysical Research 67 (1962) 423-434.

[22] A. Mishev, S. Tuohino and I. Usoskin, Neutron monitor count rate increase as a proxy for dose rate assessment at aviation altitudes during GLEs, J. Space Weather Space Clim. 8 (2018) A46.

[23] D. Cooke, J. Humble, M. Shea, D. Smart, N. Lund, I. Rasmussen, B. Byrnak, P. Goret and N. Petrou, On cosmic-ray cutoff terminology, Il Nuovo Cimento C 14 (1991), no. 3 213-234.

[24] J. Clem, Contribution of obliquely incident particles to neutron monitor counting rate, Journal of Geophysical Research 102 (1997) 919.

[25] J. Cramp, M. Duldig, E. Flückiger, J. Humble, M. Shea and D. Smart, The October 22, 1989, solar cosmic enhancement: ray an analysis the anisotropy spectral characteristics, Journal of Geophysical Research 102 (1997), no. A11 24 237-24 248.

[26] E. Vashenyuk, Y. Balabin, J. Perez-Peraza, A. Gallegos-Cruz and L. Miroshnichenko, Some features of the sources of relativistic particles at the sun in the solar cycles 21-23, Advances Space Research 38 (2006), no. 3 411-417.

[27] A. Mishev, L. Kocharov and I. Usoskin, Analysis of the ground level enhancement on 17 May 2012 using data from the global neutron monitor network, Journal of Geophysical Research 119 (2014) $670-679$.

[28] A. Mishev and I. Usoskin, Analysis of the ground level enhancements on 14 July 2000 and on 13 December 2006 using neutron monitor data, Solar Physics 291 (2016), no. 4 1225-1239.

[29] A. Mishev, S. Poluianov and I. Usoskin, Assessment of spectral and angular characteristics of sub-GLE events using the global neutron monitor network, Journal of Space Weather and Space Climate 7 (2017) A28.

[30] A. Mishev, I. Usoskin, O. Raukunen, M. Paassilta, E. Valtonen, L. Kocharov and R. Vainio, First analysis of GLE 72 event on 10 September 2017: Spectral and anisotropy characteristics, Solar Physics 293 (2018) 136.

[31] L. Desorgher, E. Flückiger, M. Gurtner, M. Moser and R. Bütikofer, A GEANT 4 code for computing the interaction of cosmic rays with the earth's atmosphere, International Journal of Modern Physics A 20 (2005), no. A11 6802-6804.

[32] J. Picone, A. Hedin, D. Drob, and A. Aikin, NRLMSISE-00 empirical model of the atmosphere: Statistical comparisons and scientific issues, Journal of Geophysical Research: Space Physics 107 (2002), no. A12 1468.

[33] I. Usoskin, G. Kovaltsov and I. Mironova, Cosmic ray induced ionization model CRAC:CRII: An extension to the upper atmosphere, Journal of Geophysical Research Atmospheres 115 (2010), no. 10 D10302.

[34] A. Mishev and P. Velinov, Normalized ionization yield function for various nuclei obtained with full Monte Carlo simulations, Advances of Space Research 48 (2011), no. 1 19-24. 